\title{
Getting a grip on aging
}

\author{
Allen R. Huang MDCM
}

Previously published at www.cmaj.ca

$\infty \quad$ See related research article by Ling and colleagues, page 429

$\mathrm{L}$ ing and colleagues offer an intriguing observation about handgrip strength and mortality in the oldest old. The authors report on their well-designed analysis of the data from the Leiden 85-plus observational cohort study from the Netherlands. ${ }^{1}$ The original cohort consisted of 599 (of the total of 705) 85-year-old inhabitants of Leiden, who were followed for 9.5 years. ${ }^{2}$ Although $80 \%$ of the cohort had died during the observational period, enough data were obtained for a reliable analysis showing an association between poor handgrip strength and mortality. How may this finding, which provides clues to the biologic behaviour of the oldest old, help us as health care clinicians?

The global population is getting older, and the fastest growing segment includes people aged 80 years and older. ${ }^{3}$ Society and health care systems need to acknowledge the rapid growth of this age group and prepare to meet its potential needs. Handgrip strength is an easy measurement for clinicians to obtain. Handgrip dynamometers, though not commonly found in physicians' offices, are simple, low-maintenance devices.

Handgrip strength has been shown to correlate closely with functional capacity in the elderly. ${ }^{4}$ As a measurable characteristic, it may reflect physical reserves and provide additional defining parameters to the concept of frailty. ${ }^{5}$ The data provided by Ling and colleagues allows normative tables for handgrip strength to be extended up to age 89 years. As a simple and reliable tool for assessment, a test of handgrip strength should be welcomed by clinicians as an additional aid to evaluating elderly patients and targeting appropriate services, procedures and interventions for them.

For instance, a patient who is under consideration for an elective surgical procedure may need to have the procedure delayed because of a weak handgrip strength on measurement, which implies that the patient's physical reserves are suboptimal. In such a scenario, an indepth functional assessment may reveal a condition for which preoperative rehabilitation could improve postoperative outcomes.

The authors report that the comorbidities and numbers of prescribed medications of study participants were equally distributed across the tertiles of handgrip strength. This observation suggests that the extent of one's reserve capacity may determine one's survival - perhaps by conferring a greater

From the Division of Geriatric Medicine, McGill University Health Centre, Royal Victoria Hospital, Montréal, Que.

CMAJ 2010. DOI:10.1503/cmaj.100004

\section{Key points}

- Poor or declining handgrip strength in the oldest old is associated with poor survival.

- Measurement of handgrip strength is easy to do, but requires a special instrument (i.e., a dynamometer).

- The addition of handgrip strength measurement to the assessment of elderly patients may help clinicians target appropriate services, procedures and interventions for them

ability to adapt to disease and disability. Hence, an accelerated decline in a person's serial handgrip strength may signal that bad things are about to happen.

The most intriguing question this study raises is whether providing interventional strength-based training to elderly people who fall below a threshold of handgrip strength would have an impact on their functional capacity and survival. For the aging person (baby boomers take heed), such studies resonate with health- and activity-conscious goals. Manufacturers of medical equipment may ultimately see an expansion in their direct-to-consumer market given the potential importance of instruments designed to measure handgrip strength.

Future studies involving populations that are more heterogenous than the Dutch cohort of Leiden may very well show different biologic behaviour among the oldest old. But the findings of Ling and colleagues could stimulate further investigation into mechanisms for successful survival as we age.

Competing interests: None declared.

\section{REFERENCES}

1. Ling CHY, Taekema D, de Craen AJM, et al. Handgrip strength and mortality in the oldest old: the Leiden 85-plus study. CMAJ 2010;182:Feb. 8. [Epub ahead of print.]

2. von Faber M, Bootsma-van der Wiel A, van Exel E, et al. Successful aging in the oldest old. Who can be characterized as successfully aged? Arch Intern Med 2001; 161:2694-700.

3. World population ageing. 1950-2050. New York (NY): United Nations Publications; 2002. p. 23. Available: www.un.org/esa/population/publications /worldageing19502050/ (accessed 2010 Jan. 25).

4. Rantanen T, Volpato S, Ferrucci L, et al. Handgrip strength and cause-specific and total mortality in older disabled women: exploring the mechanism. J Am Geriatr Soc 2003;51:636-41.

5. Fried LP, Tangen CM, Walston J, et al. Frailty in older adults: evidence for a phenotype. J Gerontol A Biol Sci Med Sci 2001;56:M146-56.

Correspondence to: Dr. Allen Huang, Rm. M8.12, Division of Geriatric Medicine, McGill University Health Centre, Royal Victoria Hospital, 687 Pine Ave. West, Montréal QC H3A 1A1; allen.huang@muhc.mcgill.ca

All editorial matter in CMAJ represents the opinions of the authors and not necessarily those of the Canadian Medical Association. 